
store of learned patterns and behaviours and introduces new positive instructions...'

'In a hypnotic state, the conscious mind is suppressed and the subconscious is released, allowing involuntary and effortless responses'

'the subconscious mind, where fears reside, is accessed in this relaxed trance state.'

'Science has agreed that hypnosis involves being in a trance state, experiencing relaxation at the same time as heightening the mind's imagination. In this state, where the subconscious is released...'

When these uninformed and erroneous statements are made, perpetuating the myths and fallacies that surround hypnosis, it is little wonder that dental professionals believe that hypnosis is all about control, trances and 'bypassing the conscious mind'. It is certainly not something to be undertaken with anxious and stressed patients, or without extensive training.

Hypnosis relies on both the power of observation and the effective use of words to evoke healing responses. When hypnosis is viewed mistakenly as a controlled altered state of conscious, people feel intimidated by the complexity and sophistication that appears to be involved.

Virtually the entire academic fraternity and research community do not adhere to the notion of hypnosis being trance or the unconscious mind as being a vast storehouse of memories and wisdom. ${ }^{1,2,3}$

These non-evidence-based statements are not a valid explanation of what is going on in hypnosis. They create a barrier of misunderstanding that prevents dental professionals from utilising the very valuable tool of hypnosis to manage anxiety and stress not only in clinical situations, but for themselves in the very physical and mental demanding profession that is dentistry.

Helping a patient reduce their anxiety about receiving dental treatment can make a huge difference to the experience for both the patient and the dental clinician. One good dental experience can then be utilised as a prototype for future dental experiences and although the patient may not eagerly await the next appointment, they don't have to fear or dread it each time.

\section{What exactly is hypnosis?}

It is an important starting point in understanding hypnosis to recognise the everpresent nature of interpersonal influence. In understanding hypnosis as a process, and not an altered state of consciousness whereby the patient is a passive subject, one realises that it is not about control or 'putting someone under'.
Hypnosis is nothing to do with trance. It is about every day cognitive faculties and how humans shape their reality. When understanding hypnosis as a cognitive process, and that it is largely a skill that can be learnt by anyone, the dental practitioner utilising hypnosis can enable the patient's subjective experience to be altered and therapeutic influence to take place. ${ }^{4}$

The building blocks for hypnosis are verbal and non-verbal communication.

The skill of empathic understanding is the foundation and the most basic ingredient in the empathic process is attending and listening to what the patient says, noticing what they do, and being fully and actively involved in the process.

Helpfully, these also are the skillsets for effective communication taught in dental schools.

The patient in hypnosis does not respond mechanically to the suggestions given, but rather in an active and goal directed manner. ${ }^{5}$

Encouraging the patient to adopt such a hypnotic mind set simply means that they will be motivated to experience it, and confident in their ability to achieve and be optimistic about the hypnosis process as a whole. awareness spontaneously, such as when daydreaming or driving a car. It is important to note that hypnotic states can be either positive or negative. When a patient enters the dental surgery where they experience overwhelming anxiety and/or stress, along with thoughts or anticipation of a painful procedure, they facilitate an internal focus of attention. This produces a hypnotic state, albeit a negative one. Hypnosis requires the patient to be engaged and focused and does not always need the state of relaxation. ${ }^{7}$ This is important to bear in mind, as it is therefore possible to refocus a patient's attention to a more positive hypnotic state.

The special communication experience that initiates a positive hypnosis state can begin in the dental surgery between the clinician and patient, with the clinician recognising the anxiety state of the patient, listening intently to the patient's explanations, and focusing on their physiology and primary sensory representational system. ${ }^{8}$

The dental clinician's role is to facilitate the process of building a positive internal focus through suggestion. By focusing intently on his or her internal experience, the patient can suspend attentiveness to the fearful external

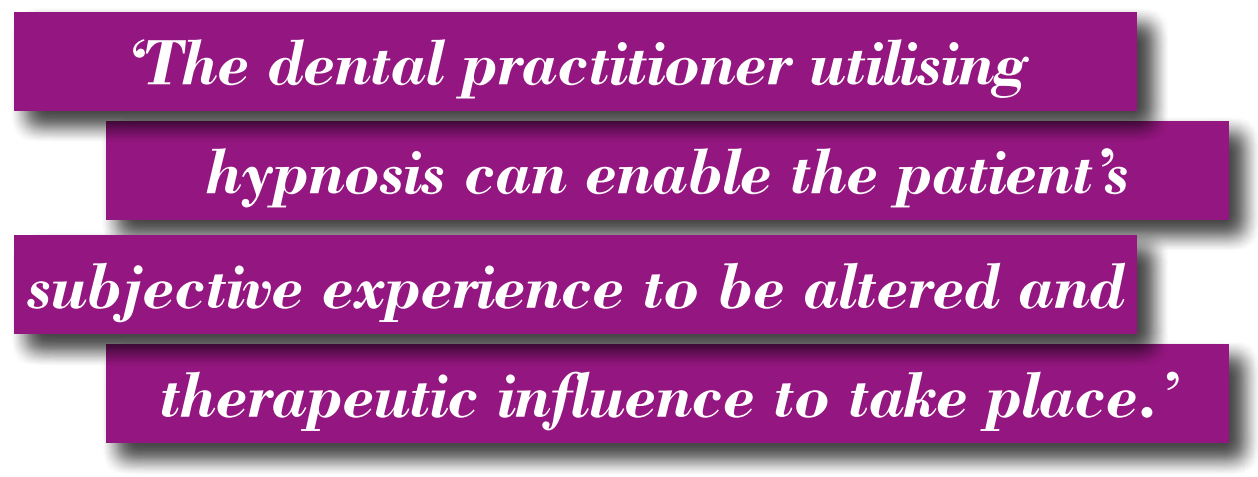

The mechanics of the experience of hypnosis include focus, belief and motivation. The importance of positive expectancy (the patient's beliefs, motivations and attitudes) cannot be emphasised enough. Kirsch (1990), suggests that the single best predictor of that behaviour is a person's positive expectancy that they are likely to produce a given behaviour (eg positive outcomes in hypnotherapy). ${ }^{6}$

The hypnotic narrowing of the focus of attention facilitates reduced awareness of unwanted stimuli from the environment such as pain and/or anxiety. This mental state enhances openness to the input of suggestions to use the imagination in order to experience changes in emotions, beliefs, physical sensations, attitudes and behaviours. ${ }^{2}$

People routinely enter conditions of experiential absorption, focus, and dissociated world, diminish reality-testing, and thereby subjectively experience a wider range of possibilities.

The relaxation techniques adopted for stressed and anxious patients are to allow them to rebalance their autonomic nervous system from the sympathetic response, to that of and access to the parasympathetic nervous system. Telling the patient to relax when they are confronted with a clinical procedure that can cause discomfort is ineffective, as the patient is not relaxed either physiologically nor emotionally. The highly anxious patient is very susceptible to signs that can help them interpret the near uncontrollable situation that they find themselves in, which is where utilising conversational hypnosis and guided imagery is useful. Such suggestions, when they come from a confident and professional figure of authority, assists the patient 
accepting the idea, or content of suggestions, as their subjective reality. They subsequently become emotionally convinced that the world is as the suggestion describes it, as the highly anxious patient in the fear state follows suggestions automatically without logic or critical evaluation. ${ }^{9}$

Patients in this modified state respond with the emotional parts of their brain, therefore it is up to the dental clinician to recognise this opportunity for intervention and choose the right words to respond to that state, as well as trust their own observation skills and intuition regarding their observations in the therapeutic process. ${ }^{10}$ hypnosis is essentially a way of organising therapeutic communications to best fit the patient's individual needs, using words and gestures selectively in order to arrive at a positive outcome for the patient - which is a positive outcome for any dental professional.

Good dental practice is communication and interpersonal influence, and that is precisely where hypnosis comes in.

The discovery of mirror neurons ${ }^{11}$ provides strong evidence in favour of longrecognised concepts of modern clinical hypnosis regarding the significance of the therapeutic relationship. Mirror neurons in our brain provide the possible neurobiological

\section{'Relaxation gives the patient the}

\section{freedom to control themselves,}

\section{especially when the underlying fear of}

\section{dental anxiety is loss of control.'}

Therefore, helping a patient reduce their anxiety about receiving dental treatment with a few well-chosen statements can make a huge difference to the outcome. Relaxation gives the patient the freedom to control themselves, especially when the underlying fear of dental anxiety is loss of control. Being able to relax and let go implies a change from anticipating the worst and dreading the unknown, to feeling secure in the knowledge that everything is under control.

As with all therapeutic interventions, hypnosis involves skilled communication between patient and clinician. The rituals of formal hypnosis inductions do not have to be incorporated in order to be hypnotic in verbal communication and demeanour in developing rapport with patients. That said, studying hypnosis offers substantial insights into how human beings construct their individual realities, and how the various intrapersonal components of human experience can be assembled to help generate wellness.

The success of hypnosis in a clinical setting requires trust between clinician and the patient to go along with the process, which reflects the trust between clinician and patient with the dental treatment itself. Indeed, the patient who feels understood by the clinician is the patient more likely to benefit from the diagnosis, treatment and education they experience as they are more likely to take suggestions on board. The approach to dental mechanism of how we resonate with one another's emotions and experience what someone else is experiencing.

The effective advantage of the mirror neurons system for hypnosis has been demonstrated by Eve Banyai's research in $1998,{ }^{12}$ where she shows there is a tendency for participants in hypnotic relationships to mirror each other's body language and autonomic psychophysiology as the hypnotic experience deepens.

\section{Why hypnosis for dentistry}

In dentistry, any treatment provided to patients should be based on sound scientific principles and a demonstrated level of clinical safety and effectiveness. In recent years, hypnosis has increasingly become the subject of serious scientific inquiry, effectively scaling down the grandiose claims of practitioners while steadily increasing what can be said in its favour with substantive evidence. ${ }^{13}$

Given its high prevalence worldwide, dental fear can be considered a universal phenomenon with different cultural features. ${ }^{14}$ Coriat (1946) emphasised the concept of dental fear as a form of anticipatory anxiety, not necessarily depending on expected pain, and defined the fear of a danger which is unknown as related to a feeling of helplessness in an anticipated traumatic situation. ${ }^{15}$

Although pain is subjective and a matter of experience and emotion, most research and clinical practice have only focused mechanistically on analgesic and anaesthetic drugs, understating its nature and forgetting the patient's role in its management. ${ }^{16,17}$

A meta-analysis for hypnotically induced analgesia ${ }^{18}$ found that for $75 \%$ of the population, hypnosis provided substantial pain relief, regardless of the type of pain that is experienced. This supports the view that hypnotically suggested analgesia meets the criteria for a 'well established treatment.' ${ }^{19}$

In terms of the subjective individual pain threshold and blood pressure, the use of hypnosis is experienced as very similar to the effect of sedation, according to a study by Fanini et al. ${ }^{20}$

Continuous dental care and a treatment relationship are the required aims of the dental profession in order to offer a good standard of service to the public. However, in spite of the increased understanding of patient management skills, dental professionals increasingly experience stress with the challenging demands in treating anxious patients.

An evaluation of dentists' perceived needs regarding the treatment of anxious patients ${ }^{21}$ found that the over-riding concern that arises from this work is that dentists feel they are not satisfactorily trained to manage anxious patients which doubtless leads to the feelings expressed of stress and lack of confidence?.

It appeared the vast majority of 


\section{Conclusion}

Most patients visit the dentist in an overwhelming state of negative expectation, and it is this expectation that plays a role in maintaining the dental anxiety, with selfverbalisation and catastrophising ensuring this negative thought feedback loop.

This heightened sense of alertness, however, can be utilised very easily to induce hypnosis. The value is in understanding that hypnosis is not relaxation in terms of decreased awareness or alertness, but it is relaxing some parts of the body and the brain to allow for focusing on others and it can be done very successfully in patients who are anxious, tense and unhappy, as they are already in this narrowed focus of attention.

Dental clinicians already aim to create rapport, or positive expectancy, by behaving and speaking confidently and inspiring confidence in patients every day of their practice life. Learning hypnosis as a cognitive tool to manage not only the patient's anxiety and pain, but also to ameliorate the stresses and challenges that are faced every day in the surgery, can change the whole perception of job management as well as manage the worklife balance. What a wonderful outcome.

respondents to the survey believe they do not have the proper skills or training to manage dentally anxious patients.

Sadly, the editorial comment went on to state that 'hypnosis is an example of a psychological method with poor empirical support for lasting effects on dental trait anxiety and skilled use requires a comparatively large amount of training?

It is important to remember that hypnosis per se is not a therapy. It is a tool that can be used as an adjunct to established therapy techniques that have proven efficacy in reducing anxiety symptoms. The studies in the earlier part of this paragraph are evidence for this.

For successful dental treatment, the patient's experience matters, the clinician's skills matter, and the relationship between them and the context in which they interact matter.

But while it is easy to presume that the dentist and patient relationship is a simple case of professional/patient dynamics, dentistpatient communication has been historically marked by anxiety on the part of the patient and occupational stress for the dentist. This latter problem is experienced not only in interpersonal exchanges, but over the course of a career in the kind of negative stereotyping of dentistry that has reduced the enjoyment of dental practice. ${ }^{22}$

\section{References}

1. Heap M, Aravind K K. Hartland's medical and dental hypnosis, $4^{\text {th }}$ edition. London: Churchill Livingstone, London, 2002.

2. Lynn S, Hallquist M N. Toward a scientifically based understanding of Milton H. Erickson's strategies and tactics: Hypnosis, response sets and common factors in psychotherapy. Contemporary Hypnosis 2004; 21: 63-78.

3. Nash M R, Barnier A J (eds). Oxford handbook of hypnosis: theory, research and practice. Oxford: Oxford University Press, 2008.

4. Sheehan P W, McConkey U M. Hypnosis and experience: The exploration of phenomena and process. Hillsdale, NJ: Erlbaum, 1982.

5. Lynn S J, Sivec H. The hypnotizable subject as creative problem-solving agent. In Fromm E, Nash M (eds). Contemporary perspectives in hypnosis research. pp 292333. New York, NY: Guilford Press, 1992.

6. Kirsch I. Changing expectations: A key to effective psychotherapy. Pacific Grove, CA: Brooks/Cole, 1990.

7. Bányai É I. Toward a socialpsychobiological model of hypnosis. In Lynn S J, Rhue J W (eds). Theories of hypnosis: Current models and perspectives. pp 564-598. New York: Guilford Press, 1991.

8. Grinder J, Bandler R. Trance-formations: neurolinguistic programming and the structure of hypnosis. Utah: Real People Press, 1981.

9. Bejenke C J. Painful medical procedures. In Barber J (ed). Hypnosis and suggestion in the treatment of pain. pp 209-265. New York \& London: Norton \& Company, 1996.

10. Barber J. Hypnosis and awareness. In Zeig J (ed). Ericksonian hypnotherapy, Volume 1: Structures. pp 245-255. New York: Brunner/ Mazel, 1985.

11. Ferrari P F, Gallese V, Rizzolatti G, Fogassi L. Mirror neurons responding to the observation of ingestive and communicative mouth actions in the monkey ventral premotor cortex. Eur J Neurosci 2003; 17: 1703-1714.

12. Banyai E I. The interactive nature of hypnosis: Research evidence for a socialpsychobiological model. Contemporary Hypnosis 1998; 15: 52-64.

13. Lynn S J, Kirsch I, Rhue J W. An introduction to clinical hypnosis. In Lynn S J, Rhue J W, Kirsch I (eds). Handbook of clinical hypnosis. pp 3-18. American Psychological Association, 2010.

14. Berggren U, Pierce C J, Eli I. Characteristics of adult dentally fearful individuals. A cross-cultural study. Eur J Oral Sci 2000; 108: $268-274$.

15. Coriat I H. Dental anxiety: fear of going to the dentist. Psychoanal Rev 1946; 33: 365-367.

16. Aydede M. Is feeling pain the perception of something? J Philosophy 2009; 106: 531-567.

17. O'Sullivan B, Schroer R. Painful reasons: Representationalism as a theory of pain. The Philosophical Quarterly 2012; 62: 737-758.

18. Montgomery G H, DuHamel K N, Redd W H. A meta-analysis of hypnotically induced analgesia: how effective is hypnosis? Int $J$ Clin Exp Hypn 2000; 48: 138-153.

19. Chambless D L, Hollon S D. Defining empirically supported therapies. J Consult Clin Psychol 1998; 66: 7-18.

20. Fanini D, Poglio M, Marci M C, Iovinelli G, Antenucci F. Oral premedication with clonidine as an alternative in dental practice. The effects on the pain threshold, blood pressure and salivary flow. Minerva Stomatol 1998; 47: 453-464.

21. Hill K B, Hainsworth J M, Burke F J T, Fairbrother K J. Evaluation of dentists' perceived needs regarding treatment of the anxious patient. Br Dent J 2008; 204: E13.

22. Jones L M, Huggins T J. Empathy in the dentist-patient relationship: review and application. NZ Dent J 2014; 110: 98-104.

https://doi.org/10.1038/s41407-021-0529-8 